\title{
OBSERVATORIO
}

\section{DERECHO E INFORMACIÓN}

\author{
César Iglesias-Rebollo
}

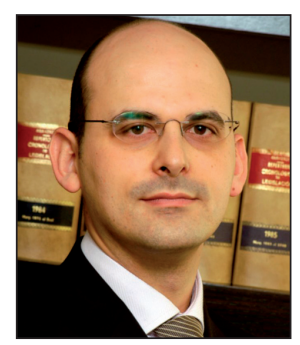

César Iglesias-Rebollo es asesor en derecho mercantil, resolución de conflictos y protección de las inversiones, protección de datos, propiedad intelectual e industrial y en derecho del entretenimiento. Su experiencia profesional se ha desarrollado en empresas públicas y privadas en el sector de la propiedad intelectual y las nuevas tecnologías, habiendo escrito diversas publicaciones en estos campos. Ha asesorado a la Comisión Europea en proyectos de open access y open data, y la Biblioteca Digital Europea. Socio fundador de la consultora empresarial Kuroshiro. Secretario general de Aseda (Asociación para el Estudio y la Enseñanza del Derecho de Autor). Miembro del claustro del Máster de abogacía internacional y del Master in sport law del Instituto Superior de Derecho y Economía (ISDE). Especialista universitario en propiedad intelectual por la Universidad Pontificia de Comillas. Diploma in legal studies por Cardiff Law School. International executive master in business administration por el Instituto de Empresa. Licenciado en economía por la UNED. Licenciado en derecho por la Universidad Pontificia de Comillas, cuenta con más de diez años de experiencia profesional.

Umer \& Co

Juan Ramón Jiménez, 8

Edif. Eurobuilding, 1-6 A. 28036 Madrid ciglesias@umerandco.com http://www.umerandco.com

\section{Resumen}

Derecho e Información son dos conceptos que se necesitan el uno al otro, aunque a veces no se comprenda la extensión de la relación que tienen entre ellos. Para hacerlo patente se ofrece una panorámica general de las principales áreas donde esta relación entre Derecho e Información es más íntima y esencial. A fin de hacer más comprensible esta visión general, y únicamente con ese fin, nos centramos en las tres funciones que el Derecho puede ejercitar respecto a la Información: ocultar, preservar y compartir. Debemos evitar el riesgo de tratar dichos temas sin tener en cuenta cómo se interrelacionan todos ellos entre sí. La buena noticia es que el Derecho lleva milenios dando respuesta a las necesidades de la sociedad ante los cambios y los retos que plantean los cambios tecnológicos y sociales y que cuenta con herramientas de sobra para hacerlo de nuevo. Para usarlas sólo necesita el apoyo de los profesionales de la información que le orienten en todos los nuevos fenómenos derivados de la información digital.

\section{Palabras clave}

Derecho, Información, Regulación, Intimidad, Protección de datos de carácter personal, Derecho al olvido, Secreto de Estado, Secretos comerciales, Información sanitaria, Derecho de autor, Creative commons, Obras huérfanas, Agujero digital, Patrimonio documental, Patentes, Open access, Open data, Ley de Transparencia.

Title: Law and information

\begin{abstract}
Law and Information are two concepts that need each other, although the complexity of their relationship is not always fully understood. We propose an overview of the main areas where this relationship is more intimate and fundamental. To make this overview more comprehensive we will focus on the three functions that Law can exercise over Information: hide, preserve and share. All topics discussed are highly related and the discussion of one has to take into account the other. The good news is that Law has been responding to the needs of the society due to the challenges posed by technological and social changes for millenia and it has the tools to do it again. For this, legal practitioners need the support of information professionals to guide them through all the new phenomena arising from digital information.
\end{abstract}

\section{Keywords}

Law, Information, Regulation, Privacy, State's secrets, Right to be forgotten, Personal data protection, Trade secrets, Health information, Copyright, Creative commons, Orphan works, digital gap, documentary heritage, patents, Open access, Open data, Spanish transparency act. 
Iglesias-Rebollo, César. "Derecho e información". El profesional de la información, 2012, septiembre-octubre, v. 21, n. 5, pp. 449-452.

http://dx.doi.org/10.3145/epi.2012.sep.01

\section{Una relación necesaria}

Derecho e Información son dos conceptos que se necesitan el uno al otro, aunque a veces no se comprenda la extensión de la relación que tienen entre sí.

Recordemos que la Información es el conocimiento obtenido por un ser humano a partir de los datos que percibe, es la base de nuestra relación con el mundo exterior y con los demás.

Por su parte, y evitando ser dogmáticos, el Derecho es el arte de la regulación de las relaciones humanas. En lo que nos importa aquí, en una sociedad moderna, dicha regulación requiere fijarse en la información que se obtiene de tres tipos de datos: los que obtiene el Estado de sus ciudadanos, los que los ciudadanos reciben del Estado y, finalmente, los datos que los ciudadanos intercambian entre ellos.

Al igual que el proceso mental de obtener información a partir de los datos es sumamente complicado, el Derecho sigue un proceso complejo para establecer la regulación adecuada a una relación determinada. En ese proceso complejo hay dos elementos que deben tenerse en cuenta para comprender cualquier regulación:

- un equilibrio óptimo entre los intereses en juego, óptimo para quien regula, y;

- una serie de presupuestos técnicos, económicos y políticos generalmente aceptados por una sociedad concreta.

No sólo es un proceso complejo sino también inestable, por eso los mismos problemas (por ejemplo, cómo debe organizarse un Estado democrático o cuáles deben ser los límites de la libertad de expresión) tienen diferentes soluciones en diferentes partes del mundo y dichas soluciones deben cambiar con los movimientos económicos y sociales.

Debido a esa complejidad e inestabilidad, el Derecho necesariamente cuenta con herramientas muy flexibles para abordar los problemas derivados de regular las relaciones humanas. Las normas, siempre apoyadas en unas premisas éticas fundamentales para ser legítimas, son un elemento imprescindible para dicha regulación. Sin embargo, sólo cobran sentido al ser aplicadas, bien a través de contratos y acuerdos, bien a través de sistemas de resolución de conflictos (aunque inicialmente se piensa en el sistema judicial como la única vía de resolución de conflictos también hay que tomar en cuenta otros sistemas, con importancia creciente, como el arbitraje y la mediación).

\section{Una relación no siempre bien comprendida desde el Derecho}

Entrando a analizar cómo trata el Derecho la información se observa que los juristas no siempre han sabido entender la relación entre los datos, la información y los documentos. Esta confusión cuando todos los datos están incorporados en documentos a los que es necesario acceder para extraer información de los mismos. No obstante, en el ámbito digital los documentos (el continente) dejan de tener relevancia, cobrando primacía los datos y la información (el contenido).

Ejemplos de esta confusión se pueden ver en normativas tan importantes como la Ley de autonomía del paciente, aprobada en el año 2002, donde todavía el legislador define la historia clínica como un "conjunto de documentos", quizás desconociendo, entre otras cosas, que información muy relevante para dicha historia clínica se almacena en bases de datos que se puede incorporar o no a documentos.

Por otro lado, la vigente regulación que prevé la sustitución de certificados en papel (Real Decreto 209/2003) habla no sólo de certificados telemáticos, esto es la emisión de documentos electrónicos equivalentes a los certificados en papel, sino también de "transmisiones de datos" para que las administraciones públicas intercambien directamente información.

Podemos hablar, por tanto, de que vivimos en una época de transición en la que el Derecho se está adaptando, al ritmo al que se adapta la propia sociedad, a la información digital. Esta transición tiene muchos frentes abiertos que habitualmente se estudian de forma aislada. Sin embargo, este enfoque fragmentado o vertical, por sectores, impide a veces ver los problemas horizontales que es necesario solucionar antes de poder avanzar. Asimismo, dada la intensidad del cambio en muchos de ellos es fácil perder la perspectiva de cómo las soluciones y problemas en uno de ellos afectan a otros.

\section{Una relación que cumple tres funciones}

Por tanto, proponemos a continuación hacer una panorámica general de las principales áreas donde esta relación entre Derecho e Información es más íntima y esencial.

A fin de hacer más comprensible esta visión, y únicamente con ese fin, vamos a entender que la relación entre Derecho e Información, para considerarse plenamente desarrollada, debe cumplir tres funciones: ocultar, preservar y compartir.

\section{Para un profesional de la información puede resultar chocante que una de las principales funciones del Derecho res- pecto a la información es ocultarla}

\section{Ocultar la información}

Para un profesional de la información puede resultar chocante que una de las principales funciones del Derecho respecto a la información es ocultarla.

No obstante, si pensamos en los flujos de información de los que hablábamos al principio veremos cómo este aspecto es fundamental para el correcto desarrollo de las relaciones en un estado democrático y de derecho.

Así, todos damos por sentado que tenemos derecho a ocultar determinada información nuestra frente a terceros, sobre 
nuestra vida personal, tales como nuestras creencias políticas y religiosas, vida sexual, etc. Esta protección impide que el Estado pueda ejercitar un control sobre los ciudadanos incompatible con la democracia. El derecho a la intimidad es el que articula esta protección de los ciudadanos frente al Estado y a otros ciudadanos. Dado el volumen de información que generamos y la facilidad de acceso a la misma, este derecho está sufriendo un fuerte ataque por la facilidad de acceder y rastrear de forma sistemática las comunicaciones e información en formato digital (llamadas, correos electrónicos, transacciones bancarias, etc.).

El derecho a la protección de datos de carácter personal cobra extrema importancia a medida que el derecho a la intimidad se va erosionando. Se trata de devolver el control de sus datos a los ciudadanos cuando éstos han sido accedidos o están custodiados por terceros.

La más reciente evolución del derecho a la protección de datos es el llamado "derecho al olvido", a dejar de hacer accesibles determinadas informaciones personales publicadas en internet. Muchas informaciones que, a pesar de ser públicas, antes quedaban enterradas bajo el peso de las resmas de papel ahora quedan expuestas a cualquiera que tenga acceso a un buscador de internet. Multas de tráfico, "pecados" de juventud fotografiados, notas de oposiciones, todo ello son datos que quedan accesibles a cualquiera que nos rastree en la Red, sin que importen los años que han pasado. Por eso en las últimas propuestas de regulación comunitaria del derecho de protección de datos de carácter personal surge este llamado "derecho al olvido".

\section{En las últimas propuestas de regulación comunitaria del derecho de protección de datos de carácter personal surge el "derecho al olvido"}

Por otro lado, la limitación del acceso por los ciudadanos a la información del Estado plantea muchas cuestiones que son difíciles de abordar en el poco espacio disponible. Para nuestros efectos, basta con recordar que el Estado cuenta con diversas regulaciones para proteger con diferentes niveles de seguridad, diferentes tipos de informaciones vinculadas con la persecución de delitos, la defensa nacional, prevención de riesgos para la seguridad nacional, etc.

En el flujo de la información entre ciudadanos entran en juego no sólo el derecho a la intimidad y el derecho a la protección de datos, ya mencionados, sino también información comercialmente valiosa (información que afecte a la competencia, secretos comerciales, información de patentes antes de la presentación de la solicitud de registro, información protegida por acuerdos de confidencialidad, etc.) así como información que afecte al honor y al uso de la propia imagen.

\section{Preservar la información}

La preservación de la información no llama la atención tanto como otras cuestiones y, sin embargo, es una función imprescindible del Derecho que puede garantizar la integridad, autoría y calidad de la información recibida. La información en un contexto digital requiere una ayuda especial del Dere- cho para su preservación porque resulta posible alterarla de un modo que no era posible hasta hace poco.

Piénsese que, así como los contenidos de una hemeroteca tradicional no son alterables. Sí resulta factible modificar los archivos digitales de una publicación para eliminar, por ejemplo, un artículo que haya sido considerado injurioso por los tribunales. En un momento que parte creciente de nuestra memoria colectiva está en formato digital ¿debemos dejar que se pueda reescribir dicha memoria?

Así, el Derecho de Autor juega aquí un papel fundamental, dado que mucha información valiosa se comparte en formatos protegidos como propiedad intelectual (por ej. un artículo científico). El Derecho de Autor da herramientas a quienes estén interesados en la preservación de la información para evitar su adulteración y manipulación. De forma paradójica, también los derechos de autor han surgido como un obstáculo para la preservación de la cultura en la medida en que han contribuido a crear una serie de incertidumbre jurídicas, como el tratamiento de las "obras huérfanas", que han obstaculizado la puesta en marcha de medidas de preservación de la cultura de nuestra época. Es el llamado "agujero digital" que debemos abordar de forma prioritaria.

En relación con lo anterior, también se plantea la necesidad de establecer sistemas de gestión de los derechos de autor de los contenidos digitales (a fin de poder reconocer y otorgar derechos sobre los mismos). El elemento más interesante de dichos sistemas es una suerte de catalogación de los objetos digitales que permita vincularlos con sus creadores. La evolución de estos sistemas permitirá identificar y rastrear los contenidos digitales y conocer sus creadores (el proyecto Arrow es un adelanto de estos sistemas).

Un área donde resulta vital la preservación de la información es en el ámbito sanitario. Tengamos en cuenta que estamos viviendo los comienzos de unos profundos cambios en los sistemas sanitarios en el que la información clínica va a estar en soporte digital. No sólo eso, sino que esa información va a ser controlada y gestionada por el propio paciente, quien va a tener una mayor influencia sobre el tratamiento de su enfermedad, apoyado en la capacidad de informarse a través de internet sobre su enfermedad. Es fundamental que esta información sea fiable y confiable. La realidad social ha dejado muy atrás la práctica jurídica y es un área en la que es necesario trabajar para preservar la salud de todos.

La información también tiene protección por vía de la legislación que regula el patrimonio histórico en el que se incluye el patrimonio documental y archivístico. La aplicación de esta regulación todavía tiene pendiente de abordar, al menos en España, los retos que plantea la información en el ámbito digital y su compatibilidad con la protección de los datos de carácter personal (o sea, con mantener oculta la información como hemos visto anteriormente) y con una mayor transparencia de las administraciones públicas (en el ánimo de compartir la información, como veremos a continuación).

\section{Compartir la información}

La preocupación que más ha llamado la atención entre los profesionales de la información en los últimos años es la de 
compartir la información. Esto es, cómo poner las condiciones para que se pueda compartir y hacer accesible la mayor cantidad de información posible.

Una fórmula jurídica ya consagrada para fomentar la publicación y compartir información de tipo científico y técnico ha sido el derecho a patentar, cuya idea es muy sencilla: el inventor recibe un monopolio de fabricación a cambio de dar información detallada sobre su invención. Es cierto que los diferentes sistemas de patentes han sido cuestionados por, según quien critique, dar demasiada protección o demasiada poca. Sin embargo, no puede olvidarse el papel fundamental del derecho de patentes en animar a los inventores a difundir y compartir sus invenciones.

Paradójicamente, la salvaguarda de los derechos de autor presenta incertidumbres jurídicas que en el caso de las "obras huérfanas" ha obstaculizado su preservación. Es el llamado "agujero digital" que debemos abordar de forma prioritaria

Con el paso del tiempo el problema del acceso a la información científica y técnica se ha trasladado a las publicaciones científicas. El movimiento Open Access (OA) propugna una cambio de modelos de negocio y de políticas de publicación para garantizar que los artículos científicos sean accesibles a todos. El OA plantea retos desde el punto de vista económico y de política de desarrollo científico. No obstante, el Derecho es una palanca para el cambio o un obstáculo para recorrer los caminos del OA (sean del color que sean).

Un elemento importante, como no podía ser de otra ma- nera, en el desarrollo del OA pero también para cualquier plataforma de intercambio y puesta a disposición de contenidos son las cuestiones relativas al derecho de autor. En este sentido, las licencias Creative commons han permitido dar una respuesta sencilla, cómoda y estandarizada a la necesidad de otorgar autorizaciones para el uso de contenidos protegidos por el derecho de autor.

Un área en la que España lleva retraso y en la que sólo ahora parece empezar a ponerse al día es la del acceso a la información de las administraciones públicas. Recientemente se ha aprobado el proyecto de ley de la llamada Ley de transparencia, que confiemos que llegue a buen puerto. La iniciativa europea Open Data defiende que la información de las Administraciones Públicas debe ser accesible a todos los ciudadanos para que la puedan reutilizar como estimen conveniente, buscando, por un lado, las externalidades positivas de compartir información y, por otro, las ventajas de todo tipo que trae para una sociedad democrática una administración pública más abierta y transparente.

\section{Una relación con futuro}

Este recorrido nos ha permitido recordar que Derecho e Información son palabras que van juntas y que están destinadas a entenderse en relación con muchos temas. Debemos evitar el riesgo de tratar dichos temas sin tener en cuenta cómo se interrelacionan todas ellos. La buena noticia es que el Derecho lleva milenios dando respuesta a las necesidades de la sociedad ante los cambios y los retos que plantean los cambios tecnológicos y sociales y que cuenta con herramientas de sobra para hacerlo de nuevo. Para usarlas sólo necesita el apoyo de los profesionales de la información que le orienten en todos los nuevos fenómenos derivados de la información digital.

\title{
Próximos temas centrales
}

Noviembre 2012

Enero 2013

Marzo 2013

Mayo 2013

Julio 2013

Septiembre 2013

\section{Bibliotecas académicas}

\section{Soportes digitales}

\author{
Educación y biblioteca
}

Bibliotecas y documentación de museos

Economía de la información

\section{Gestión de contenidos}

Los interesados por favor consulten detenidamente las Normas para autores: http://www.elprofesionaldelainformacion.com/autores.html

y luego envíen sus artículos a través del gestor de manuscritos OJS de la plataforma del Recyt: http://recyt.fecyt.es/index.php/EPI/index 